\title{
New Measurements of Deconvoluted EDS Spectra of Elemental Standards as a Function of Atomic Number and Electron Beam Energy.
}

\author{
M.S. Hatzistergos and E. Lifshin
}

College of Nanoscale Science and Engineering, University at Albany, 251 Fuller Road, Albany NY 12203

In electron probe microanalysis (EPMA) composition is calculated from experimental K-ratios by applying various correction procedures such as ZAF and $\varphi(\rho z)$. The K-ratio is defined as the ratio of background and deadtime corrected intensity measured from a given specimen to that of a similarly corrected measurement from a bulk standard. Generally, in conventional EPMA standard intensities are acquired at the time of each analysis. In so called "standardless" analysis, standard intensities are determined from adjusted stored data possibly used in conjunction with a theoretical model. However, according to Newbury et al., results based on "standardless" analysis can be subject to large errors ranging from $-90 \%$ to $+150 \%$ [1]. Therefore, any effort to improve our ability to accurately estimate standard intensities will help improve "standardless" analysis $[2,3]$. The present work was undertaken to address the basic question of how does X-ray emission from standards vary with atomic number and beam energy. Furthermore, calibration curves derived from the results can be used to estimate standard intensities over a wide range of experimental conditions.

Thirty seven pure elemental standards were used in this study, ranging in atomic numbers from $12(\mathrm{Mg})$ to $92(\mathrm{U})$. The beam energies used were 5, 10, 15 and $20 \mathrm{keV}$ and the beam current ranged from 0.7 to $6.5 \mathrm{nA}$. X-ray spectra were collected in a JEOL 8600 electron microprobe (thermionic emission gun) equipped with an EDAX EDS detector at a take off angle of $40^{\circ}$. A Faraday cup was used to measure the beam current before and after each measurement. Live time of 100 seconds was used for each measurement and the dead time was kept below 25\%. After background subtraction, the spectral region containing characteristic peaks was fitted to a model consisting of multiple Gaussian peaks, the number of which depended on the element. To reduce the number of fitting parameters the FWHM of K lines (no interference) was used as a reference to compute the FWHM of $\mathrm{L}$ and $\mathrm{M}$ lines using the expression of Fiori and Newbury [4]. Knowing the Gaussian parameters of each peak and treating X-ray lines that differ less than $\sim 15 \mathrm{eV}$ as one peak, the net counts of each line was determined by adding the number of counts in channels at $\pm 3 \sigma$ around the peak. The average number of counts over three measurements was between 10,000 and 150,000 for the major peaks, ensuring good statistics (relative standard deviation generally $<1 \%$ ). Figures 1a-c show representative plots of $\mathrm{K} \alpha, \mathrm{L} \alpha$ and $\mathrm{M} \alpha$ measured intensities (cts/nAsec) as a function of atomic number. Such curves are also available for other K, L and M-series lines since deconvolution of the spectra provided net counts for all lines. Work is in progress to find an analytical expression relating the data and explain the scattering observed in the M-lines measurements.

A study of X-ray intensity of several representative elements was also performed as a function of beam energy. The beam energies ranged from 5 to $20 \mathrm{keV}$ in increments of $2 \mathrm{keV}$. Although no attempt was made to calculate the detector's solid angle and hence determine absolute efficiencies, the measured intensities were transformed to generated intensities by correcting for sample absorption by: $\mathrm{I}_{\text {emitted }} / \mathrm{I}_{\text {generated }}=\mathrm{f}(\chi)=(1+\alpha \gamma \chi)^{-2}[5]$. The detector absorption was not a significant factor for the range of studied energies. Figure 1d shows a plot of the generated intensity 
(cts/nAsec) versus beam energy $(\mathrm{keV})$. The data seem to follow a function of the form $A(U-1)^{\mathrm{n}}$, where $U$ is the overvoltage ratio [2].

The findings of this work are being used to refine existing Monte Carlo simulation programs, specifically the one by Gauvin et al., and thus further improve the accuracy in predicting X-ray spectra [6]. This will allow the analyst to calculate K-ratios for cases where standards are not available, select optimal experimental conditions, calculate detection limits and analyze non conventional samples such as thin films and particles.

References

[1] D.E. Newbury, C. R. Swyt and R.L. Myklebust, Analytical Chemistry (1995) 67(11), 1866.

[2] M.S. Prasad and D.C. Joy, Scanning (2003) 25(4), 210.

[3] D.E. Newbury, R.L. Myklebust and C.R. Swyt, Microbeam Analysis (1995) 4(4), 221.

[4] C.E. Fiori and D.E. Newbury, Scanning Electron Microscopy (1978) 1, 401.

[5] K.F.J Heinrich and H. Yakowitz, Analytical Chemistry (1975) 47(14), 2408.

[6] R. Gauvin, E. Lifshin et al., Microscopy and Microanalysis (2006) 12, 1.
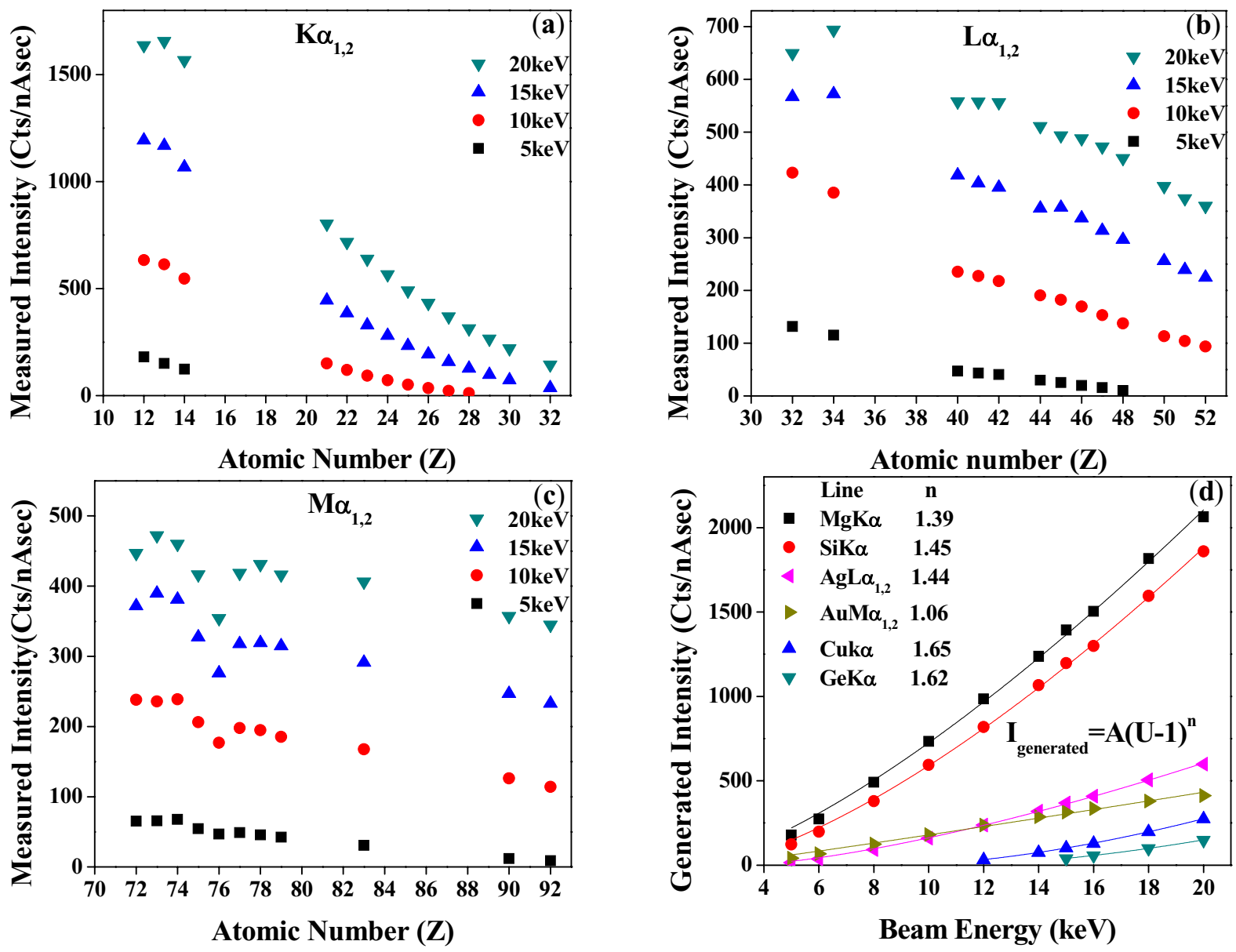

FIG. 1. Measured intensity vs atomic number of (a) $K \alpha_{1,2}$, (b) $L \alpha_{1,2}$ and (c) $M \alpha_{1,2}$ lines. (d) Generated intensity vs beam energy and fit to $A(U-1)^{n}$. 